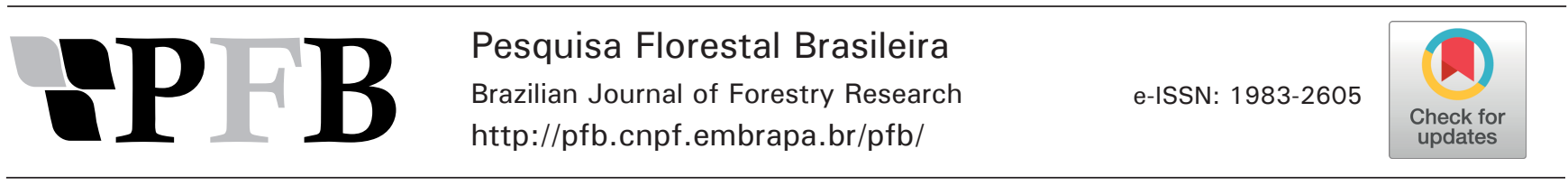

\title{
Estádio inicial da regeneração natural de espécies arbóreas em área alterada no Estado do Amazonas
}

\author{
Wanda Lemos Paixão Nogueira ${ }^{1}$ (D), Marciel José Ferreira ${ }^{1 *(D)}$, Narrúbia Oliveira de Almeida Martins ${ }^{1}$ (D) \\ Universidade Federal do Amazonas, Av. Rodrigo Octávio Jordão Ramos, 6200, Coroado, CEP 69080-900, Manaus, AM, Brasil
}

*Autor correspondente:

mjf.ufam@gmail.com

Termos para indexação:

Sucessão secundária

Composição florística

Espécies pioneiras

Index terms:

Secondary succession

Floristic composition

Pioneer species

Histórico do artigo:

Recebido em 14/05/2016

Aprovado em 21/05/2018

Publicado em 11/10/2018

$\underline{\text { doi: } 10.4336 / 2018 . p f b .38 \mathrm{e} 201601265}$
Resumo - O melhor entendimento da composição florística e estrutura da regeneração natural, em diferentes tipos de distúrbio, é fundamental para identificar o grau de recuperação da área e possíveis intervenções silviculturais a serem eventualmente adotadas para acelerar o processo de sucessão florestal. O presente estudo objetivou caracterizar a regeneração natural de 0,4 ha em processo de recuperação natural na Fazenda Experimental da Universidade Federal do Amazonas na expectativa de identificar o potencial da condução da regeneração para a recuperação da área. Foram estabelecidas cinco parcelas de $15 \mathrm{~m}$ x $9 \mathrm{~m}$ e amostradas as plântulas e os indivíduos arbóreos jovens com altura superior a $10 \mathrm{~cm}$. Foram registrados 148 indivíduos pertencentes a oito famílias botânicas, nove gêneros e 13 espécies arbóreas. Após 14 meses sem interferências, a área foi dominada por espécies do gênero Vismia, as quais se destacaram em grande parte nos parâmetros estruturais (densidade, dominância, frequência, valores de importância e cobertura). O sítio estudado possui regeneração natural com baixa diversidade florística, dominada por indivíduos de espécies predominantemente pioneiras, que pode estar associado ao uso anterior com cultivo agrícola, ao pequeno intervalo de tempo sem interfência e à forte competição com espécies de plantas daninhas.

\section{Initial stage of natural regeneration of tree species in a disturbed area in Amazonas State, Brazil}

\begin{abstract}
The better understanding of floristic composition and structure of natural regeneration in differents types of disturbances is essential to identify the degree of recovery of the area and silvicultural interventions to be eventually adopted in order to accelerate the process of forest sucession. The present study aimed to characterize the natural regeneration of 0.4 ha in the natural recovery process in the Experimental Farm of the Federal University of Amazonas aiming to identify the potential of natural regeneration tendency for recovering the area. Tree saplings with height bigger than 10 $\mathrm{cm}$ were sampled in five plots of $15 \mathrm{~m}$ x $9 \mathrm{~m}$. We registered 148 seedlings and saplings from eight botanical families, nine genera and thirteen tree species. After 14 months without interference, the area was dominated by species of the genus Vismia, which had the highest values of the most structural parameters (density, dominance, frequency, importance and coverage values). The studied site presents natural regeneration with low floristic diversity, dominated by individuals of pioneer species, which may be associated with the previews use as agriculture land, the short period since the last interference and the strong competition with grasses species.
\end{abstract}




\section{Introdução}

O Bioma Amazônia acumula atualmente redução de cerca de 720 mil km² de fitofisionomias florestais (Fonseca et al., 2015). Os efeitos negativos deste impacto são sentidos em todo o planeta, com consequências bem mais amplas e severas quando comparado aos impactos causados pelo desflorestamento de quaisquer outras áreas de florestas tropicais no mundo (Lawrence \& Vandecar 2015).

Mesmo considerando o histórico de desaceleração nas taxas de desflorestamento desde o nível máximo alcançado em 2004 (Godar, 2014), o constante surgimento de novas áreas desflorestadas e as baixas taxas de revegetação dessas áreas na Amazônia, revela que o desflorestamento e os impactos negativos associados a ele estão longe de serem controlados (Godar, 2014; Instituto Nacional de Pesquisas Espaciais, 2016; Fonseca et al., 2015).

No cenário atual de conversão da floresta para outras formas de uso do solo, estima-se que cerca de 172.000 $\mathrm{km}^{2}$ das áreas que sofreram desflorestamento encontra-se em processo avançado de regeneração, embora outros $97.000 \mathrm{~km}^{2}$ ainda sejam classificados como pasto sujo ou em fase inicial de regeneração, sendo, portanto, passíveis de ações para a condução da regeneração. A expectativa é que, se recuperadas, essas áreas minimizariam em $37 \%$ os índices de áreas degradadas, as quais têm sido utilizadas para fins de reflorestamento em apenas $0,42 \%$ de sua extensão total (Instituto Nacional de Pesquisas Espaciais, 2016).

Diante do potencial da regeneração natural das áreas alteradas, a sua condução com o objetivo de reabilitar áreas que perderam sua cobertura florestal seria uma opção viável, principalmente por ser um método menos oneroso, uma vez que elimina os custos associados à mão de obra e insumos para a produção de mudas e plantio (Shono et al., 2007).

Apesar da possibilidade de uso da condução da regeneração natural como método de recomposição vegetal de áreas alteradas, é importante considerar as limitações desse método, com destaque para sua restrição a ambientes pouco degradados e com alto grau de resiliência, mantendo condições propícias à germinação das sementes presentes nos solos e ao crescimento das mudas estabelecidas naturalmente (Lamb et al., 2005; Holl \& Aide, 2011). O sucesso do método depende ainda do isolamento da área para a eliminação dos fatores de degradação e de espécies competidoras, bem como de ações para reduzir barreiras que possam dificultar o estabelecimento da regeneração natural, tais como a compactação do solo e a baixa disponibilidade de fontes de propágulos (Venturoli et al., 2011; Souza et al., 2012). Ainda assim, mesmo que esses pressupostos sejam atendidos, é possível que em longo prazo as espécies regenerantes não garantam a sucessão ecológica, caso a composição florística seja predominantemente de espécies pioneiras de ciclo de vida curto, não ocorrendo espécies dos estágios mais avançados da sucessão ou o recrutamento de novas espécies vegetais por meio do banco de sementes do solo, dispersão de sementes, rebrotamento de troncos danificados ou regeneração adiantada (Lamb et al., 2005; Venturoli et al., 2011; Souza et al., 2012).

Diante do papel importante da composição vegetal para se definir o sucesso da condução da regeneração natural, o objetivo desse estudo foi analisar a composição florística e a estrutura da regeneração natural de espécies arbóreas de uma área alterada, pela perda da sua cobertura vegetal, e verificar o potencial deste método para o reestabelecimento da vegetação na área. Ao considerar o curto período sem interferências (14 meses), a expectativa é que o método de regeneração natural tenha potencial para a revegetação da área, porém o estágio de sucessão se encontre em fase inicial, com domínio de espécies do grupo ecológico das pioneiras.

\section{Material e métodos}

\section{Localização e caracterização da área de estudo}

O estudo foi conduzido em uma área de 0,4 ha localizada na Fazenda Experimental da Universidade Federal do Amazonas (FAEXP-UFAM), nas coordenadas 2³9'19,3”S e 6003'09,6”W, Manaus, AM. O clima da região é tropical quente e úmido (tipo Amw, segundo a classificação de Köppen), com umidade relativa do ar entre $84 \%$ e $90 \%$, precipitação média anual de $2.100 \mathrm{~mm}$ e $25{ }^{\circ} \mathrm{C}$ a $28{ }^{\circ} \mathrm{C}$ de temperatura média. A vegetação original era composta de floresta tropical úmida de baixa altitude (Ribeiro et al., 1999).

A área de estudo possui relevo levemente acidentado, com solo predominantemente do tipo Latossolo Álico argiloso, ácido e pobre em nutrientes (Chauvel, 1982), fazendo parte de um conjunto de áreas na FAEXP destinadas à condução de experimentos agrícolas, de 
pecuária, de pesca, florestais entre outros. Previamente à instalação do experimento, a floresta foi cortada, a área foi cultivada com mandioca e posteriormente abandonada (Figura 1A). No entanto, não há registro do tempo exato de uso da área após a remoção da cobertura florestal. A regeneração natural das espécies arbóreas foi analisada após 14 meses sem interferências na área (Figura 1B). Áreas de florestas secundárias dominadas por indivíduos dos gêneros Bellucia, Goupia, Vismia e Cecropia e plantios adultos do gênero Parkia estão localizadas ao lado da área do experimento.
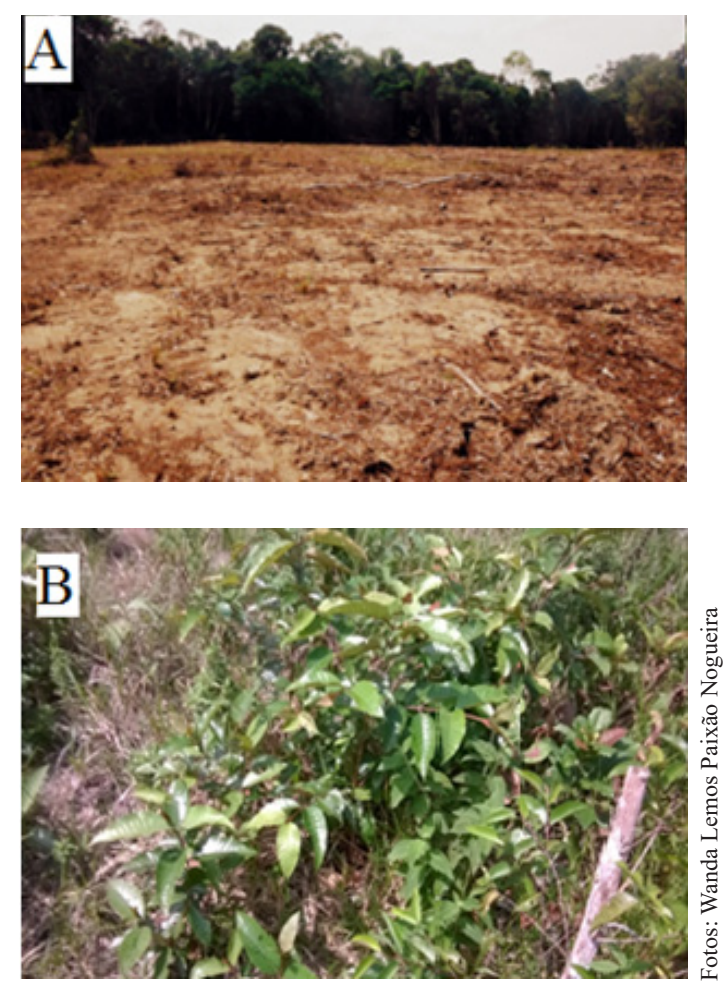

Figura 1. A) Área inicialmente alterada na Fazenda Experimental da Universidade Federal do Amazonas (FAEXP-UFAM). B) Regeneração natural da área após 14 meses sem interferências.

Figure 1. A) Site initially disturbed in the Experimental Farm of the Federal University of Amazonas (UFAMFAEXP). B) Natural regeneration of the area after 14 months without disturbance.

\section{Análise da composição florística e estrutura da regeneração natural}

Para a caracterização da regeneração natural, foram instaladas cinco unidades amostrais com tamanho de $9 \mathrm{~m} \times 15 \mathrm{~m}$, resultando em uma área total de amostragem de $675 \mathrm{~m}^{2}$ (cerca de $0,07 \mathrm{ha}$ ) ou $17 \%$ da área total do experimento ( 0,4 ha). Quatorze meses após o isolamento da área, foi realizado um inventário da regeneração natural dentro de cada uma das parcelas.

Todos os indivíduos arbóreos regenerantes com altura superior a $10 \mathrm{~cm}$ foram identificados quanto à família e espécie, com o auxílio de um parabotânico, considerando o sistema Cronquist, sendo medidos seus respectivos valores de altura e diâmetro à altura do solo (DAS), para posterior categorização em diferentes classes de tamanho, (adaptado de Pereira et al., 2014): Classe I - plantas com altura entre 0,1 e $0,3 \mathrm{~m}$; Classe II - plantas com altura superior a $0,3 \mathrm{~m}$ e inferior a 1,5 m; Classe III - plantas com altura entre 1,5 e 3,0 m.

A estrutura horizontal foi analisada por meio dos parâmetros frequência, densidade e dominância, em valores absolutos e relativos e das classes de tamanho da regeneração natural. A partir desses resultados, foram calculados: valor de importância (VI), valor de cobertura (VC), índice de regeneração, classes de tamanho da regeneração natural, índice de diversidade de Shannon (H') e índice de equabilidade de Pielou (J'), conforme descrito em Freitas \& Magalhães (2012). Os cálculos foram processados em planilha eletrônica.

\section{Resultados e discussão}

No inventário florístico da regeneração natural foram encontrados 148 indivíduos, distribuídos entre 8 famílias botânicas, 9 gêneros e 13 espécies arbóreas (Tabela 1). A família botânica com maior abundância foi Clusiaceae, representada por 4 espécies, com $85 \%$ dos indivíduos pertencentes ao gênero Vismia (Figura 1B).

Os gêneros Vismia e Cecropia têm sido os mais abundantes em estudos de sucessão secundária de áreas alteradas na região amazônica (Araujo et al., 2009; Massoca et al., 2012; Longworth et al., 2014; Williamson et al.; 2014). Todavia, ambientes onde a regeneração natural é dominada por Vismia não seguem o modelo clássico de sucessão ecológica (Jakovac et al., 2012), uma vez que independente da idade da floresta secundária dominada por estas espécies, os povoamentos possuem um padrão de arquitetura de copa mais baixo e com folhages mais espessas, promovendo maior entrada de luz no ambiente, o que favorece a permanência dos indivíduos das espécies pioneiras, limitando assim o recrutamento de espécies tolerantes à sombra e consequentemente o avanço do processo sucessional (Jakovac et al., 2012). Por outro 
lado, povoamentos de Cecropia com o avanço da idade aumentam a interceptação de luz, podendo promover mais rapidamente a recuperação de espécies da floresta madura tolerantes à sombra.

A alta dominância de determinadas espécies na composição florística tem sido comum em ambientes recém - perturbados (Souza et al., 2010), principalmente pelo fato desses ambientes serem dominados por gramíneas, as quais limitam a presença de outras espécies (Klippel et al., 2015). Espécies arbóreas pioneiras típicas (e.g. Cecropia spp. e Vismia spp.) são mais eficientes no uso de recursos primários, tais como luz, água e nutrientes, sobressaindo nos estádios iniciais sobre as demais espécies florestais (Silva et al., 2008, 2011).

Tabela 1. Relação das espécies arbóreas amostradas na regeneração natural em área alterada no Amazonas, em ordem alfabética de famílias e espécies, seguidas dos seus respectivos números de indivíduos.

Table 1. List of tree species sampled of natural regeneration from a disturbed site in Amazonas State, in alphabetical order of families and species, followed by their respective number of trees.

\begin{tabular}{|c|c|c|c|c|c|c|c|}
\hline Família/Espécie & GE & $P_{1}$ & $\mathbf{P}_{2}$ & $\mathbf{P}_{3}$ & $\mathbf{P}_{4}$ & $\mathbf{P}_{5}$ & $\begin{array}{c}\mathbf{N}^{0} \text { de } \\
\text { Indivíduos }\end{array}$ \\
\hline \multicolumn{8}{|l|}{ Anacardiaceae (1) } \\
\hline Tapirira guianensis & $\mathrm{P}$ & & 1 & & & 1 & 2 \\
\hline \multicolumn{8}{|l|}{ Flacourtiaceae (1) } \\
\hline Casearia sylvestris & $\mathrm{P}$ & & & & & 1 & 1 \\
\hline \multicolumn{8}{|l|}{ Clusiaceae (4) } \\
\hline Vismia cayennensis & $\mathrm{P}$ & & & 37 & 2 & & 39 \\
\hline V. gracilis & $\mathrm{P}$ & & 14 & 21 & 12 & 9 & 56 \\
\hline V. guianensis & $\mathrm{P}$ & 1 & 3 & & 8 & 19 & 31 \\
\hline V. japurensis & $\mathrm{P}$ & & & 1 & & & 1 \\
\hline \multicolumn{8}{|l|}{ Mimosoideae (1) } \\
\hline Pentachlera macroloba & $\mathrm{NP}$ & & & 2 & & 1 & 3 \\
\hline \multicolumn{8}{|l|}{ Melastomataceae (2) } \\
\hline Bellucia dichotoma & $P$ & & 1 & & & & 1 \\
\hline Miconia alata & $P$ & & 2 & 2 & & & 4 \\
\hline \multicolumn{8}{|l|}{ Memecylaceae (1) } \\
\hline Mouriri sp. & $\mathrm{NP}$ & & & & & 1 & 1 \\
\hline \multicolumn{8}{|l|}{ Solanaceae (1) } \\
\hline Solanum guianensis & $\mathrm{P}$ & 1 & & & 1 & & 2 \\
\hline \multicolumn{8}{|l|}{ Urticaceae (2) } \\
\hline Cecropia sciadophylla & $\mathrm{P}$ & & & 1 & & & 1 \\
\hline Cecropia distachya & $\mathrm{P}$ & & 1 & 5 & & & 6 \\
\hline Total de indivíduos & & 2 & 2 & 69 & 23 & 32 & 148 \\
\hline
\end{tabular}

$\mathrm{P}_{\mathrm{n}}=$ parcela $\mathrm{n}$; grupo ecológico $=\mathrm{GE} ; \mathrm{P}=$ pioneira; $\mathrm{NP}=$ não pioneira.

Em relação à distribuição das espécies é possível inferir que poucas espécies contribuem de maneira efetiva para a estrutura fitossociológica da regeneração natural, uma vez que a abundância de indivíduos é representada, predominantemente, pelas espécies $V$. gracilis (38\%), V. caynensis (26\%) e V. guianensis (21\%), as quais constituem cerca de $85 \%$ do total de indivíduos amostrados (Tabela 2). Dentre as espécies mais frequentes, apenas as espécies $V$. gracilis e $V$. caynensis estiveram presentes em $80 \%$ das parcelas alocadas na área de estudo, as quais também estiveram entre as espécies mais abundantes e dominantes na regeneração, 
indicando assim a importância fitossociológica dessas espécies para a estrutura da vegetação.

Embora C. distachya tenha apresentado altos valores de frequência e dominância, esta teve baixa densidade, quando comparada às espécies do gênero Vismia, indicando que o parâmetro que mais influenciou para definir o valor de importância das espécies neste estudo foi a densidade das espécies em regeneração.
Além de alcançar o maior valor de importância e de cobertura, uma vez que estes indicadores consideram os parâmetros densidade, frequência e dominância para expressar a importância de cada espécie na regeneração natural, $V$. gracilis também se destacou em relação ao índice de recuperação natural, o que também considera a distribuição das espécies nas diferentes classes de tamanho da regeneração natural (Freitas \& Magalhães, 2012).

Tabela 2. Estrutura horizontal e classes de tamanho da regeneração natural de espécies arbóreas em área alterada no Amazonas.

Table 2. Forest structure and size classes of natural regeneration of tree species from a disturbed site in Amazonas State.

\begin{tabular}{lcccccccccccc}
\hline \multicolumn{1}{c}{ Família } & Espécie & DA & DR & FA & FR & DoA & DoR & VI & VC & CTARN & CTRRN & RN \\
\hline Clusiaceae & Vismia gracilis & 830 & 37,8 & 80 & 16 & 0,04 & 41,4 & 95,3 & 79,3 & 5567 & 39,2 & 31,00 \\
Clusiaceae & Vismia cayennensis & 578 & 26,4 & 40 & 8 & 0,03 & 23,4 & 57,8 & 49,8 & 3540 & 24,9 & 19,75 \\
Clusiaceae & Vismia guianensis & 459 & 20,9 & 80 & 16 & 0,01 & 8,8 & 45,7 & 29,7 & 2612 & 18,4 & 18,44 \\
Urticaceae & Cecropia distachya & 88,9 & 4,05 & 40 & 8 & 0,01 & 10,8 & 22,9 & 14,9 & 587 & 4,1 & 5,39 \\
Anacardiaceae & Tapirira guianensis & 29,6 & 1,35 & 40 & 8 & 0,01 & 5,3 & 14,7 & 6,7 & 238 & 1,7 & 3,68 \\
Mimosoideae & Pentachlera macroloba & 44,4 & 2,03 & 40 & 8 & 0,0 & 3,4 & 13,4 & 5,4 & 354 & 2,5 & 4,17 \\
Melastomataceae & Miconia alata & 59,3 & 2,7 & 40 & 8 & 0,0 & 2 & 12,7 & 4,7 & 470 & 3,3 & 4,67 \\
Solanaceae & Solanum guianensis & 29,6 & 1,35 & 40 & 8 & 0,0 & 2,5 & 11,9 & 3,9 & 238 & 1,7 & 3,68 \\
Melastomataceae & Bellucia dichotoma & 14,8 & 0,68 & 20 & 4 & 0,0 & 0,6 & 5,3 & 1,3 & 122 & 0,9 & 1,84 \\
Urticaceae & Cecropia sciadophylla & 14,8 & 0,68 & 20 & 4 & 0,0 & 0,5 & 5,2 & 1,2 & 122 & 0,9 & 1,84 \\
Flacortiaceae & Casearia sylvestris & 14,8 & 0,68 & 20 & 4 & 0,0 & 0,5 & 5,2 & 1,2 & 122 & 0,9 & 1,84 \\
Clusiaceae & Vismia japurenses & 14,8 & 0,68 & 20 & 4 & 0,0 & 0,4 & 5,1 & 1,1 & 122 & 0,9 & 1,84 \\
Memecylaceae & Mouriri sp. & 14,8 & 0,68 & 20 & 4 & 0,0 & 0,4 & 5,1 & 1,1 & 122 & 0,9 & 1,84 \\
\hline & & 2193 & 100 & 500 & 100 & 0,1 & 100 & 300 & 200 & 9641 & 100 & 100 \\
\hline
\end{tabular}

Onde: $\mathrm{DA}=$ densidade absoluta; $\mathrm{DR} \%=$ densidade relativa; $\mathrm{FA}=$ frequência absoluta; $\mathrm{FR} \%$ = frequência relativa; DoA = dominância absoluta; DoR $\%=$ dominância relativa; $\mathrm{VI} \%$ = valor de importância; $\mathrm{VC} \%$ = valor de cobertura; $\mathrm{CTARN}=$ classe de tamanho absoluta da regeneração natural; $\mathrm{CTRRN} \%=$ classe de tamanho relativa da regeneração natural; RN\% = índice de regeneração natural.

No que diz respeito à distribuição das espécies nas diferentes classes de tamanho da regeneração natural, observa-se que $78 \%$ do total de indivíduos estão concentrados na classe C2 (Figura 2). V. gracilis foi a única espécie a apresentar indivíduos distribuídos em todas as classes de tamanho. Tal fato indica o potencial dessa espécie em colonizar áreas desflorestadas e abandonadas e sugere a capacidade desta espécie em persistir na comunidade ao longo do processo sucessional, contribuindo em médio e longo prazos para os processos ecológicos do sítio em regeneração (Pereira et al., 2001; Silva et al., 2007).

$V$. cayennensis e $V$. guianensis também apresentaram alto índice de regeneração natural. Porém, com a distribuição dos indivíduos limitada às classes de tamanho $\mathrm{C} 1$ e $\mathrm{C} 2$, sugerindo que a colonização por esses indivíduos é mais recente. A abundância destes na classe $\mathrm{C} 1$ indica a ocorrência de novos recrutamentos dessas espécies na regeneração natural.

Em paralelo à dominância das espécies pioneiras, também foi observado menor número de indivíduos de espécies não pioneiras (e. g. P. macroloba e Mouriri sp.). Porém, o fato da presença desse grupo ecológico estar restrita à classe $\mathrm{C} 2$ sugere não ocorrência de novas entradas desses indivíduos na regeneração natural. Este resultado pode ser explicado pela presença de espécies competidoras na área de estudo (Paspalum sp., Scoparia dulcis e Davilla sp.), dificultando o estabelecimento das plantas de regeneração natural. A presença de espécies herbáceas e/ou gramíneas típicas dos estádios iniciais da regeneração, também conhecidas como espécies invasoras agressivas (Silva et al., 2016), e que possuem 
grande capacidade de resistir à ambientes inóspitos e competir por água e nutrientes, comprometem a sobrevivência e o crescimento inicial de espécies florestais menos tolerantes, em especial aquelas dos estádios mais avançados da sucessão (Klippel et al., 2015). Assim, o sucesso do método de regeneração natural não depende apenas da presença de fontes de propágulos no entorno da área, sendo a eliminação do fator de competição fundamental para a continuidade da regeneração natural de espécies florestais (Lamb et al., 2005; Shono et al., 2007, Chazdon, 2012, Carvalho et al., 2016).

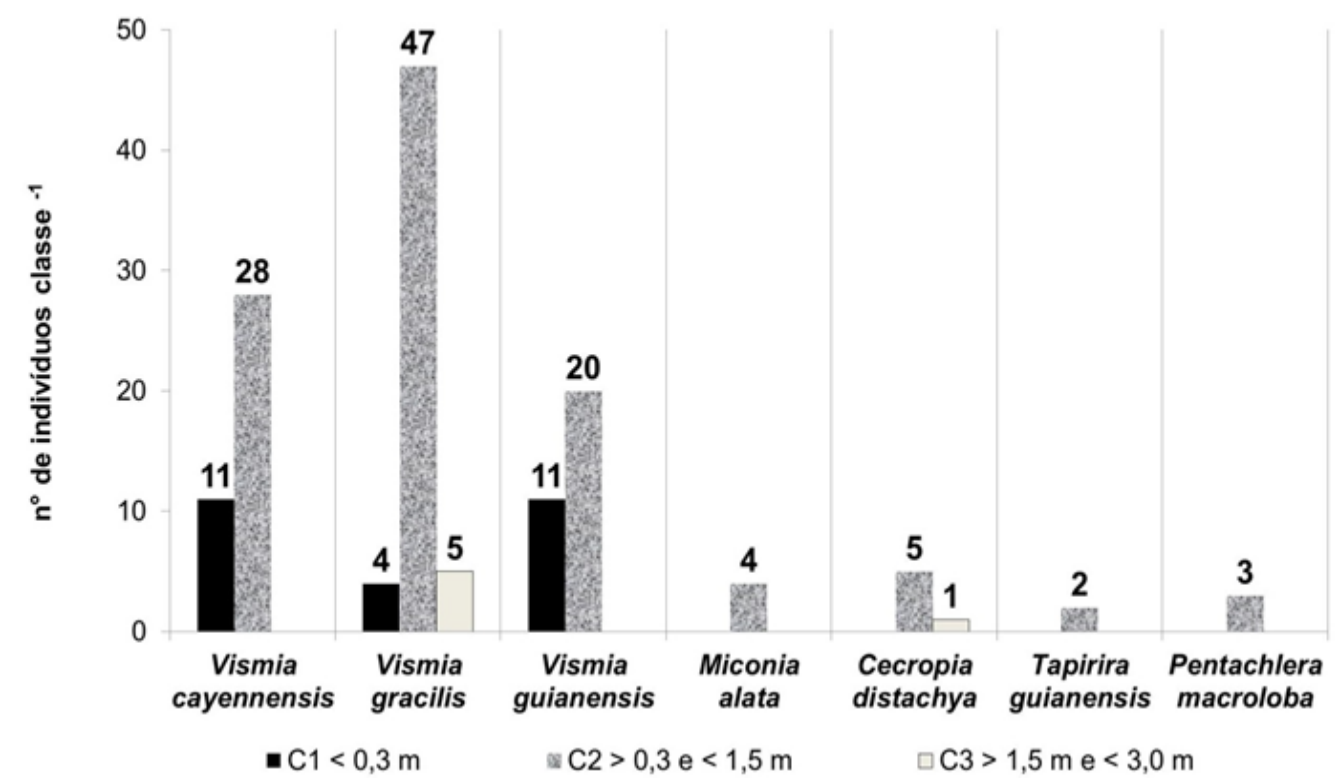

Figura 2. Densidade das sete espécies de maior índice de regeneração natural nas diferentes classes de tamanho. $\mathrm{C} 1=$ plantas com altura menor que $0,3 \mathrm{~m} ; \mathrm{C} 2=$ plantas com altura entre 0,3 e $1,5 \mathrm{~m}$ e $\mathrm{C} 3=$ plantas com altura maior ou igual $1,5 \mathrm{~m}$.

Figure 2. Density of the seven species with the highest natural regeneration index in the different size classes. $\mathrm{C} 1=$ plants with height less than $0.3 \mathrm{~m} ; \mathrm{C} 2=$ plants with height between 0.3 and $1.5 \mathrm{~m}$ and $\mathrm{C} 3$ $=$ plants with height greater or equal of $1.5 \mathrm{~m}$.

Embora os valores de diversidade de espécies da regeneração natural para florestas tropicais variem entre 3,83 nats ind. ${ }^{-1}$ e 5,85 nats ind ${ }^{-1}$ (Lima et al., 2012), a competição entre a regeneração natural de espécies arbóreas com plantas herbáceas e arbóreas típicas dos estádios iniciais, associada ao uso da área para cultivo agrícola e o tempo sem interferência na área relativamente curto, podem ter sido decisivos para o baixo valor do índice de diversidade florística observado nesse estudo $\left(\mathrm{H}^{\prime}=1,59\right.$ nats ind $\left.^{-1}\right)$. A diversidade de espécies, bem como a trajetória sucessional de uma área alterada, também pode ser influenciada por outros fatores, como pela disponibilidade e distância de fontes de propágulos (Holl \& Aide, 2011; Chazdon, 2012).

Ao quantificar a riqueza e a diversidade de espécies em áreas de florestas tropicais abandonadas após dois anos e com diferentes históricos de uso, ZermeñoHernández et al. (2015) constataram que o índice de Shannon (H') variou entre 1,0 e 3,0, estando a maioria das áreas com índice abaixo de 2,0. Esta baixa diversidade foi justificada pelos autores principalmente pela baixa disponibilidade de propágulos, fortemente reduzida em campos abertos sujeitos a longos períodos de uso agrícola. Daí a importância na manutenção de fragmentos florestais para o maior potencial de estabelecimento da regeneração natural como método de revegetação dessas áreas (Holl \& Aide, 2011; ZermeñoHernández et al., 2015).

Ao avaliar a regeneração natural de nascentes após nove anos sem intervenção, Silva et al. (2016) observaram índice de diversidade de Shannon (H') igual a 1,88 nats ind ${ }^{-1}$, não sendo observada evolução da regeneração mesmo após o enriquecimento. A baixa divesidade foi influenciada pela forte competição com espécies herbáceas e arbóreas típicas dos estádios iniciais, tidas como invasoras. Os autores sugeriram que 
para um melhor estabelecimento da regeneração natural, a mesma deve ser conduzida sob intervenção humana com práticas de manutenção da área, tais como capinas, roçadas e coroamento dos indivíduos regenerantes.

$\mathrm{O}$ valor do índice de equabilidade de Pielou (J') observado neste estudo $(0,60)$ está abaixo dos valores obtidos em outras pesquisas realizadas na Amazônia (0,75 a 0,92) (Alves \& Miranda, 2008), porém são semelhantes a outros estudos que analisaram áreas em processo de regeneração (Zermeño-Hernández et al., 2015; Silva et al., 2016). O baixo valor deste parâmetro pode ser explicado pelo grande número de indivíduos em regeneração distribuídos entre poucas espécies, predominantemente do gênero Vismia (Pinto et al., 2005; Do Vale et al., 2014).

\section{Conclusões}

O sítio estudado possui baixa diversidade florística, sendo representada predominantemente por espécies pioneiras típicas, em especial do gênero Vismia.

Os resultados configuram estádio inicial de sucessão ecológica, o qual pode estar associado à intensidade do distúrbio, ao histórico de curto período sem interferência na área e à forte competição com plantas herbáceas e arbóreas típicas dos estádios iniciais da regeneração.

A ocorrência de espécies não pioneiras provenientes de fragmentos florestais próximos à área indica o potencial de recuperação da vegetação em longo prazo, por meio do método de condução da regeneração natural, devendo-se, contudo, adotar técnicas de manejo de plantas daninhas e futuros plantios de enriquecimento de espécies dos estádios mais avançados da sucessão, como intervenções silviculturais complementares para a revegetação da área.

\section{Referências}

Alves, J. C. Z. O. \& Miranda, I. S. Análise da estrutura de comunidades arbóreas de uma floresta amazônica de Terra Firme aplicada ao manejo florestal. Acta Amazonica, v. 38, n. 4, p. 657 666, 2008. DOI: 10.1590/S0044-59672008000400008.

Araujo, R. A. et al. Florística e estrutura de fragmento florestal em área de transição na Amazônia Matogrossense no município de Sinop. Acta Amazonica, v. 39, n. 4, p. 865-877, 2009. DOI: 10.1590/ S0044-59672009000400015.

Carvalho, J. et al. Regeneração natural em povoamentos de Araucaria angustifolia e Pinus sp. em Tunas do Paraná, PR. Pesquisa Florestal Brasileira, v. 36, n. 85, p. 9-16, 2016. DOI: 10.4336/2016. pfb.36.85.892.
Chauvel, A. Os latossolos amarelos, álicos, argilosos dentro dos ecossistemas das bacias experimentais do INPA e da região vizinha. Acta Amazonica, v. 12, n. 3, p. 47-60, 1982. DOI: 10.1590/1809$43921982123 \mathrm{~S} 047$.

Chazdon, R. Regeneração de florestas tropicais. Boletim do Museu Paraense Emílio Goeldi Ciencias Naturais, v. 7, n. 3, p. 195-218, 2012.

Do Vale, I. et al. Espécies indicadas para a recomposição da floresta ciliar da sub-bacia do rio Peixe-boi, Pará. Ciência Florestal, v. 24, n. 3, p. 573-582, 2014. DOI: 10.1590/1980-509820142403006.

Fonseca, A. et al. Boletim do desmatamento da Amazônia Legal (janeiro de 2015) SAD. Belém: Imazon. 2015. Disponível em: $<$ http://imazon.org.br/PDFimazon/Portugues/transparencia_florestal/ amazonia_legal/SAD-Janeiro2015.pdf>. Acesso em: 6 jun. 2015.

Freitas, W. K. \& Magalhães, L. M. S. Métodos e parâmetros para estudo da vegetação com ênfase no estrato arbóreo. Revista Floresta e Ambiente, v.19, n. 4, p. 520-540, 2012. DOI: 10.4322/ FLORAM.2012.054.

Godar, J. et al. Actor-specific contributions to the deforestation slowdown in the Brazilian Amazon. Proceedings of the National Academy of Science of the USA, v. 43, p.15.591-15.596, 2014. DOI:10.1073/PNAS.1322825111.

Holl, K. D. \& Aide, T. M. When and where to actively restore ecosystems? Forest Ecology and Management, v. 261 p. 1558 1563, 2011. DOI:10.1016/J.FORECO.2010.07.004DOI:10.1016/J. FORECO.2010.07.004.

INSTITUTO NACIONAL DE PESQUISAS ESPACIAIS (INPE). Projeto Terraclass - Mapeamento da vegetação secundária para a Amazônia Legal; 2016. Disponível em: https://ainfo.cnptia.embrapa. $\mathrm{br} /$ digital/bitstream/item/152807/1/TerraClass.pdf

Jakovac, A. C. C. et al. Age and light effects on seedling growth in two alternative secondary successions in central Amazonia. Plant Ecology \& Diversity, v.7, p. 349-358. 2012. DOI:10.1080/17550 874.2012.716088.

Klippel, V. H. et al.de restauração florestal de Mata de TabuleirosES. Revista Árvore, v. 39, n.1, p.69-79, 2015. DOI: 10.1590/010067622015000100007.

Lamb, D. et al. Restoration of degraded tropical forest landscapes. Science, v. 310 n. 5754, p. 1628-1632, 2005. DOI: $10.1126 /$ SCIENCE. 1111773 .

Lawrence, D. \& Vandecar, K. Effects of tropical deforestation on climate and agriculture. Nature Climate Change, v. 5, p. 27-36, 2015. DOI:10.1038/NCLIMATE2430.

Lima, R. B. A et al. Fitossociologia de um trecho de floresta ombrófila densa na Reserva de Desenvolvimento Sustentável Uacari, Carauari, Amazonas, Scientia Plena, v. 8, n. 1, p.1-12, 2012. DOI:10.5039/ AGRARIA.V7I3A934.

Longworth, J. B. et al. Shifts in Dominance and Species Assemblages over Two Decades in Alternative Successions in Central Amazonia. Biotropica, v. 46, n. 5, p. 529-537, 2014. DOI:10.1111/BTP.12143.

Massoca, P. E. S. et al. Dinâmica e trajetórias da sucessão secundária na Amazônia central. Boletim do Museu Paraense Emílio Goeldi.

Ciências Naturais, v. 7, n. 3, p. 235-250, 2012. 
Pereira, I. M. et al. Estrutura fitossociológica da regeneração natural de uma área de cerrado no município de Curvelo, MG. Enciclopédia Biosfera, Centro Científico Conhecer, v. 10, n. 18, p. 1619-1636, 2014.

Pereira, I. M. et al. Regeneração natural em um remanescente de caatinga sob diferentes níveis de perturbação, no agreste paraibano. Acta Botânica Brasílica, v. 15, n. 3, p. 431-426, 2001. DOI: $10.1590 / \mathrm{S} 0102-33062001000300010$.

Pinto, L. V. A. et al. Caracterização física da bacia hidrográfica do ribeirão Santa Cruz, Lavras, MG e uso conflitante da terra em suas áreas de preservação permanente. Cerne, v. 11, n. 1, p. 49-60, 2005.

Ribeiro, J. E. L. S. et al. Flora da Reserva Ducke: guia de identificação das plantas vasculares de uma floresta de terra-firme na Amazônia Central. Manaus: INPA, 1999. 816 p.

Shono, K. et al. Application of assisted natural regeneration to restore degraded Tropical Forestlands. Restoration Ecology, Tucson, v. 15, n. 4, p.620-626. 2007. DOI: 10.1111/J.1526-100X.2007.00274.X.

Silva, C. E. M. et al. Photosynthetic traits and water use of tree species growing on abandoned pasture in different periods of precipitation in Amazonia. Photosynthetica, v. 49, n. 2, p. 246-252, 2011. DOI: 10.1007/S11099-011-0033-Z.

Silva, C. E. M. et al. Water-use efficiency of tree species following calcium and phosphorus application on an abandoned pasture, Central Amazonia, Brazil. Environmental and Experimental Botany, v. 64, n. 2, p.189-195, 2008. DOI: 10.1016/J.ENVEXPBOT.2008.03.001.
Silva, R. G. et al. Avaliação do processo de restauração de área de preservação permanente degradada no sul de Minas Gerais. Revista em Agronegócio e Meio Ambiente, v. 9, n. 1, p. 147-162, 2016. DOI: 10.17765/2176-9168.2016V9N1P147-162.

Silva, W. C. et al. Estudo da regeneração natural de espécies arbóreas em fragmento Floresta Ombrófila Densa, Mata das Galinhas, no Município de Catende, Zona da Mata Sul de Pernambuco. Ciência Florestal, v. 17, n. 4, p. 321-331, 2007. DOI: 10.5902/198050981964.

Souza, C. R. de et al. Comportamento de espécies florestais em plantios a pleno sol e em faixas de enriquecimento de capoeira na Amazônia. Acta Amazonica, v. 40, n. 1, p. 27-134, 2010. DOI: 10.1590/S0044-59672010000100016.

Souza, L. M. et al. Potencial da regeneração natural como método de restauração do entorno de nascente perturbada. Cerne, v. 18, n. 4, p. 128-138, 2012. DOI: 10.1590/S0104-77602012000400006.

Venturoli, F. et al. Avaliação temporal da regeneração natural em uma floresta estacional semidecídua secundária, em Pirenópolis, Goiás. Revista Árvore, v. 35, v. 3, p. 473-483, 2011. DOI: 10.1590/ S0100-67622011000300010.

Williamson, G. B. Convergence and divergence in alternative successional pathways in Central Amazonia. Plant Ecology \& Diversity, n. 7, v. 1, p. 341-348, 2014. DOI:10.1080/ 17550874.2012 .735714$.

Zermeño-Hernández, I. et al. Ecological disturbance regimes caused by agricultural land uses and their effects on tropical forest regeneration. Applied Vegetation Science, n. 18, v. 3, p. 443-455, 2015. DOI: 10.1111/AVSC.12161. 